\title{
Notes on Operations
}

\section{Managing Acquisitions in a Changing Environment: From Coping To Comfort}

\section{Cynthia Gozzi}

\begin{abstract}
A
cquisitions departments, like other parts of technical services, have been forced by a variety of internal and external forces to change dramatically in recent years, and there is every indication that the trend will continue and even accelerate in the future. The forces are many, and include the merging of acquisitions, cataloging, and sometimes interlibrary borrowing; a migration to team-based management; greater use of flexible work assignments; greater attention to workflow and cost/benefit analysis and redesign; and the building of partnerships with utilities and vendors to develop new services and experiment with outsourcing options. The challenges and pressures on managers and staff alike to accommodate or take advantage of these changes and add new services, while maintaining the existing ones, are real and sometimes overwhelming. All too often the only liferaft thrown to them is a crash course in stress management, when what would help most are programs to aid the understanding of how to cope with a process of change that has no foreseeable end; how to maintain a feeling of worth, security, motivation; and how to maintain control when the familiar is being questioned, fractured, rearranged, or replaced.

During a well-attended and lively program presented on Monday, July 8, 1996,
\end{abstract}

at the American Library Association's Annual Conference in New York the topic of managing a constantly changing acquisitions environment was explored. The program was sponsored by the Library Administration and Management Association's Systems and Services Section, Acquisitions Systems Committee, cosponsored by the Association for Library Collections and Technical Services Acquisitions Section's Acquisitions Discussion Group, and generously supported by Academic Book Center.

What inspired me to develop this conference program and publish the papers from it was the management challenge associated with entering a period of pervasive and dramatic change in the J. Murrey Atkins Library at the University of North Carolina at Charlottte. The changes began with the merger in 1993 of the Library, Media Services, and Computing Services organizations under the leadership of an associate vice-chancellor for library and information services (the former library director). They accelerated with the funding of a major library expansion and renovation project to be completed in 1999. Both of these events became catalysts for a major restructuring of programs and services, with changes in position for many staff members, in supervision, in coworkers, and in job responsi-

CrnTHIA I. (TIA) GozZI (cigozzi@email.uncc.edu) is Director of the J. Murrey Atkins Library, University of North Carolina at Charlotte. This introduction was originally presented at the ALA Annual Conference, July 8, 1996, New York City. Manuscript received November 8, 1996; accepted for publication January 30, 1997. 
bilities for many others. Simultaneously, we undertook the review and revision of most core processes. While the effects have been felt throughout the organization-not just in Technical Services-the latter is now operating in a significantly altered environment. These changes include:

- Two previously very separate departments (Acquisitions and Cataloging) have been combined into an integrated Technical Services unit.

- The management structure has been flattened significantly.

- Positions have been transferred out of Technical Services to other parts of the organization.

- The technical infrastructure has been reviewed and expanded.

- The outsourcing of some functions is being explored.

- Service and support expectations of Technical Services have been reviewed and redefined.

- Communication channels to and from other units have been strengthened.

While the changes are still unfolding, we know that along the way we have employed some managerial techniques that we will use again in the future and others that we will not repeat or will alter. Techniques that have worked well include:

- A series of workshops offered by Dr. Buch of the Psychology Department on coping with change,

- The open weekly sessions at which senior managers share information, answer questions, and listen to concerns,

- Updates in the library's electronic newsletter and via the listserv,

- The open door policy of the Human Resources manager, which has made her available to discuss changes and concerns one-on-one,

- Celebrations of significant milestones,

- Consultations between the leadership and external experts to plan and facilitate the change process,

- The use of a number of task forces to gather staff input and critique suggested changes,

- A genuine effort to match people with new jobs and tasks in ways that best utilize individual skills, interests, and needs,

- Significant investments in training,

- Attention to classification issues resulting from changes in responsibilities,

- Allowing support staff to be in the forefront of the change processes, and

- The conscious use of a new "language" to describe new concepts.

In retrospect, we should have recognized sooner some things that did not advance our cause in the right direction or inhibited progress. We placed too much focus on the structural aspects of change and too little on the cultural aspects. We did too little and we did it too slowly-in other words, we proceeded too cautiously and prolonged uncertainties unnecessarily. In the old culture, resistance had been used effectively to block change, and firm enough messages were not sent that, this time, it would no longer obtain the desired result. Altogether, we underestimated the impact of the old culture. We did not involve the rank-and-file staff as early as we should have, and we had progressed quite a bit into the changes before we developed an effective schedule and mode for communicating with staff. In addition, our promotion efforts for making the changes were neither aggressive nor upbeat enough-they should have projected more excitement, more creativity, and more fun.

The keynote address for the conference program was delivered by Kim Buch, an organizational psychologist and associate professor from the University of North Carolina at Charlotte, and the final presentation was by David Goble from North Carolina State University Library. Papers based on these two presentations have been published in Library Administration and Management, vol. 11, no. 3, Summer 1997. Between these two addresses were four short presentations by four acquisitions librarians who described the changes that have taken place in the Acquisitions Departments of their libraries in recent years. These four case studies are the basis for the following articles in which the writers summarize the changes that occurred and describe what they perceive as the most positive and negative aspects for the acquisitions 
staff, what the institution did to help staff adjust, and what advice they would give others in similar circumstances.

Without exception the program presenters emphasised that change is not a fleeting phenomenon from which we will emerge, either to return to the nostalgic predictability of times past or to enter a new period of welcome calm. It behooves all of us, therefore, to benefit from the experiences of our colleagues and to consider their advice. As one point of departure for continuing the dialogue, we present the following four real-life adventures of colleagues Douglas Duchin from Baruch College Library, Peter Kingsley from New York University, Randy Call from Detroit Public Library, and Christian Boissonnas from Cornell. I suspect we can all put our feet in their shoes.

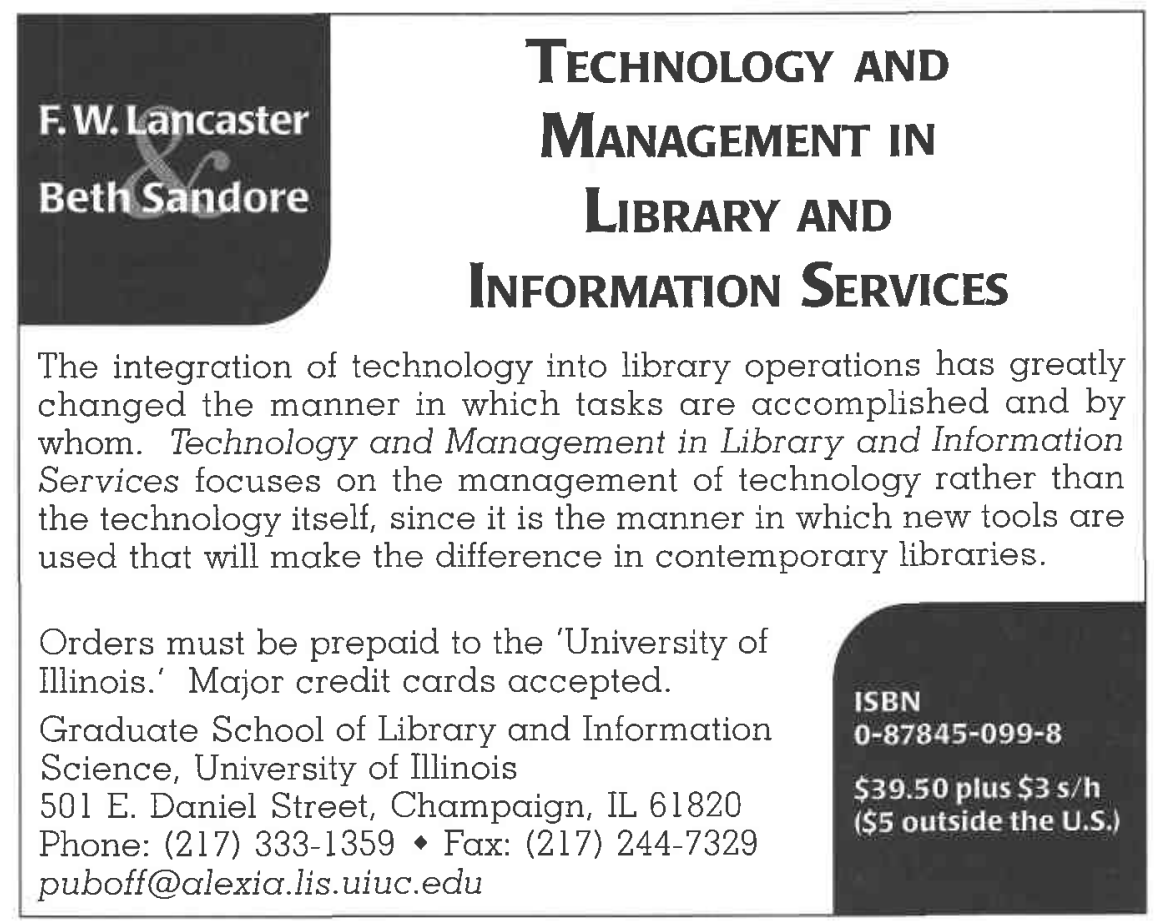

